\title{
Clinical interests of the study of adaptive oxidative / nitrosative stress in breast and ovarian cancer before and under chemotherapy- A case control Study-
}

\author{
Type of article: Conference abstract
}

Naima Badid1*, Hafida Merzouk1, Kaouel Meguenni2, Amine Charef3, Djalloul Hamzaoui4 1. Physiopthology and Biochemistry of Nutrition Laboratory (PPABIONUT), Department of Biology, Faculty of Faculty of Sciences of Nature and Life \& Sciences of the Earth and the Universe, University of Tlemcen, Algeria.

2.Epidemiology Service-University Hospital Center- Tlemcen, Algeria. 3.Oncology Service- Hospital Popular Establishment (EPH) - Maghnia - Algeria. 4.Surgery Clinic Avicene, Maghnia, Algeria.

*Corresponding author email: badidnaima@gmail.com

\begin{abstract}
:
Background: Breast and ovarian cancer are most common female cancer in Algeria in terms of incidence and mortality. Cancer cells are exposed to higher reactive oxygen species (ROS) whose levels support death evasion, angiogenesis, and metastasis. Less interest has been given to changes ROS homeostasis in cancer therapy. In this study, we investigate redox homeostasis before and after treatment (BT, AT), to determine detrimental or beneficial outcomes in cancer therapy.

Methods: Cancer patients were recruited at the Hospital of Maghnia with the engaging of healthy controls. Serum biochemical parameters and oxidant/antioxidant markers were determined.
\end{abstract}

Results:Our findings showed oxidative stress (OS) reflected by an increase in malondialdehyde (MDA), carbonyl proteins $(\mathrm{CP})$, superoxide anion $\left(\mathrm{O}_{2}{ }^{-}\right)$, nitric oxide $\left(\mathrm{NO}^{\circ}\right)$ and peroxynitrite $\left(\mathrm{ONOO}^{-}\right)$levels and a decrease in vitamins $\mathrm{C}$ and glutathione $(\mathrm{GSH})$, catalase, and superoxide dismutase (SOD) activities in cancer patients BT. After treatment (AT), levels of $\mathrm{MDA}, \mathrm{CP}, \mathrm{O}_{2}{ }^{--}, \mathrm{NO}^{\circ}, \mathrm{ONOO}^{-}$were maintained high and/or increase vis-à-vis patients BT. The lowered activities of SOD, catalase and GHS level BT, heightened in cancer cases AT. Cholesterol, triglycerides and uric acid levels were increased in BC. Uric acid levels were markedly reduced in OC patients. HDL-cholesterol levels were significantly reduced in both cancer patients.

Conclusion: Metabolic perturbations occurred with oxidative stress which highlights an adaptive appearance vis-à-vis of treatments. As a double-edged sword, redoxsignalling markers may represent a crucial point and could be the future targets for anticancer drug research.

Key words: Breast cancer; Ovarian cancer; Oxidative stress; Treatment response; Anticancer drugs.

\section{Acknowledgments}

The authors are grateful to the Directors of the Popular Hospital Establishment and the private clinic for surgery "Avicène", Maghnia, Algeria for the help in the patient recruitments for the study. They also thank all healthy volunteers for their participation to the study. 
Medical Technologies Journal, Volume: 4, Issue: 2, April-June 2020, Pages:556-557. Doi :

https://doi.org/10.26415/2572-004X-vol4iss2p556-557

\section{Conflict of interest statement}

This article is a conference abstract presented at the International Congress on Health Sciences and Medical Technologies, Tlemcen Algeria 5-7 December 2019, ICHSMT'19.

\section{Authors' biography}

No Biography.

\section{References}

No references 